\title{
Исследование глубоких центров в микроплазменных каналах фосфидгаллиевых светодиодов зеленого спектра излучения
}

\author{
(C) В.К. Ионычев, А.А. Шестеркина \\ Мордовский государственный университет, \\ 430000 Саранск, Россия \\ E-mail: microelektro@mail.ru
}

(Получена 16 фревраля 2016 г. Принята к печати 27 июля 2016 г.)

\begin{abstract}
Проведено исследование статистической задержки микроплазменного пробоя в фосфидгаллиевых светодиодах зеленого спектра излучения. В температурном диапазоне $300-380 \mathrm{~K}$ обнаружено необычное сильное влияние глубоких центров на статистическую задержку лавинного пробоя при изменении их зарядового состояния снижением обратного напряжения на $p-n$-переходе. Выявлено четыре глубоких уровня и определены их параметры.
\end{abstract}

DOI: 10.21883/FTP.2017.03.44213.8208

\section{1. Введение}

В реальных $p-n$-переходах лавинный пробой обычно сильно локализован и имеет микроплазменный характер. Микроплазмы (МП), как правило, возникают в местах скопления различных структурных дефектов и несовершенств. Характеристики и надежность полупроводниковых приборов в большой степени зависят от свойств различных дефектов и определяются совокупностью МП диода.

При приложении к $p-n$-переходу обратного напряжения, превышающего напряжение пробоя самой низковольтной МП, наблюдается задержка пробоя [1]. При большой концентрации глубоких центров (ГЦ) проявляется релаксационная задержка пробоя [2]. Если концентрация ГЦ мала, то эмиссия носителей заряда с них влияет на статистическую задержку микроплазменного пробоя [3]. В отличие от известных традиционных методов (емкостная спектроскопия, эффект Холла, фотопроводимость и т.д.), задержка лавинного пробоя дает возможность изучения характеристик локальных мест скопления дефектов, что очень важно для прогнозирования свойств и надежности полупроводниковых приборов.

Ранее было предпринято несколько попыток определения параметров ГЦ по статистической задержке лавинного пробоя [4-6]. Однако, как отмечали сами авторы, полученные результаты оказались неоднозначными и противоречивыми.

Цель данной работы - апробация метода микроплазменной спектроскопии глубоких уровней применительно к GaP:N-светодиодам, позволяющего исследовать глубокие уровни (ГУ) в микроплазменных каналах $p-n$-переходов, которые могут отвечать за процессы безызлучательной рекомбинации.

Изменение зарядового состояния ГЦ проводилось частичным снижением обратного напряжения на $p-n$ переходе. В этом случае ГЦ заполняются основными носителями заряда. При этом заполнение ГЦ проводится лишь в ограниченной части области пространственного заряда (ОПЗ) вблизи ее границ, где напряженность электрического поля относительно небольшая. Рассматривается возможность определения параметров ГЦ из измерений функции распределения длительности статистической задержки пробоя МП (кривой Лауэ) в фосфидгаллиевых светодиодах зеленого свечения.

Ранее были проведены исследования в фосфидгаллиевых светодиодах красного спектра излучения [3] и кремниевых диодах [7].

Из анализа работ $[3,7,8]$ можно сделать вывод, что данный метод обладает очень высокой чувствительностью к концентрации ГЦ по сравнению с другими методами, в частности, чувствительность даже выше, чем в Q-DLTS.

\section{2. Методика эксперимента}

В работе, как наиболее эффективный, выбран импульсный метод исследования задержки лавинного пробоя $p-n$-перехода [3]. Структурная схема измерительной установки представлена на рис. 1.

Источник постоянного напряжения (1) предназначен для питания узлов генератора (2), который вырабатывает прямоугольные импульсы напряжения с плавной регулировкой амплитуды от 0 до $300 \mathrm{~B}$ как верхнего, так и нижнего уровней. Длительность импульсов регулируется от нескольких микросекунд до десятков минут. Уровень шумов на выходе генератора (2) не превышает $0.5 \mathrm{MB}$, точность поддержания напряжения не хуже $1 \mathrm{MB}$. Длительность верхнего и нижнего уровня напряжения задаются независимо, причем длительность верхнего уровня может также определяться длительностью задержки лавинного пробоя $p-n$-перехода. Длительность фронта нарастания ступеньки напряжения не превышает 1 мкс. Все виды управления генератором прямоугольных импульсов осуществляются с помощью блока управления (6). Исследуемый образец размещается в светонепроницаемом криотермостате. Он позволяет изменять температуру образца в пределах $77-380 \mathrm{~K}$ и поддерживать ее с точностью $\pm 0.1 \mathrm{~K}$. Для точного определения температуры используется платиновый термометр сопротивления ТСПН-5. 
Подробная теория метода приведена в [3]. Например, пусть глубокий уровень находится в верхней половине запрещенной зоны и заполнение данного уровня производится основными носителями заряда (электронами) со стороны $n$-области при кратковременном снижении обратного напряжения на $p-n$-переходе. Длительность ступеньки напряжения заполнения определялась временем установления стационарного состояния в микроплазменном канале. Для уровня, расположенного в верхней половине запрещенной зоны, как правило, коэффициент эмиссии электронов с ГЦ намного больше коэффициента эмиссии дырок $e_{n} \gg e_{p}$. Поэтому в начальный момент времени $(t=0)$, после скачка напряжения $V>V_{M}$, где $V_{M}-$ напряжение пробоя микроплазмы, глубокие центры в области заполнения попадают в ОПЗ и оказываются полностью заполненными электронами. Функция распределения задержки пробоя микроплазмы по длительности (вероятность того, что в течение времени $t$ после подачи пробивного напряжения микроплазма не включится) в полулогарифмической системе координат (1) состоит из линейной (2) и нелинейной (3) частей

$$
\begin{gathered}
\ln \left(1-P_{M}\right)=\ln \left(1-P_{M 0}\right)+\ln \left(1-P_{M t}\right) \\
\ln \left(1-P_{M 0}\right)=-S_{M} N_{t} \frac{e_{n} e_{p}}{e_{n}+e_{p}}\left[\int_{-L_{p}}^{L_{n}}\left(P_{n}(x)+P_{p}(x)\right) d x\right] t \\
\ln \left(1-P_{M t}\right)=-S_{M} N_{t} \frac{e_{n}^{2}}{\left(e_{n}+e_{p}\right)^{2}} \\
\times\left[\int_{L_{m}}^{L_{n}} P_{n}(x) d x-\frac{e_{p}}{e_{n}} \int_{L_{m}}^{L_{n}} P_{p}(x) d x\right]\left[1-\exp \left(-\frac{t}{\tau}\right)\right],
\end{gathered}
$$

где $P_{M}-$ вероятность включения микроплазмы после приложения перенапряжения $\left(V-V_{M}\right), e_{n}$ и $e_{p}-$ соответственно коэффициенты эмиссии электронов и дырок с ГЦ, $P_{n}$ и $P_{p}-$ вероятности запуска ими лавины, $N_{t}-$ концентрация ГЦ, $S_{M}-$ сечение микроплазменного канала, $\tau=\left(e_{n}+e_{p}\right)^{-1}-$ постоянная времени установления стационарного состояния ГЦ, $L_{p}, L_{n}-$ границы ОПЗ $p$-n-перехода со стороны $p$ - и $n$-областей соответственно, $L_{m}$ - граница ОПЗ со стороны $n$-области при напряжении заполнения $V_{m}$ глубоких уровней.

Типичный вид обсуждаемых функций распределения представлен на рис. 2.

Линейный член (2) отражает термогенерационный механизм запуска лавины через рассматриваемый глубокий уровень. Нелинейный член (3) - запуск лавины, обеспечиваемый реэмиссией носителей с ГЦ.

Линейный член можно исключить из рассмотрения, если измерить функцию распределения без заполнения ГЦ (фоновый запуск лавины). В этом случае при малых перенапряжениях $L_{m} \approx L_{n}$ и измеряется $1-P_{M 0}$. Таким образом, задачу можно свести к анализу функции $1-P_{M t}$, содержащей основную информацию о влиянии

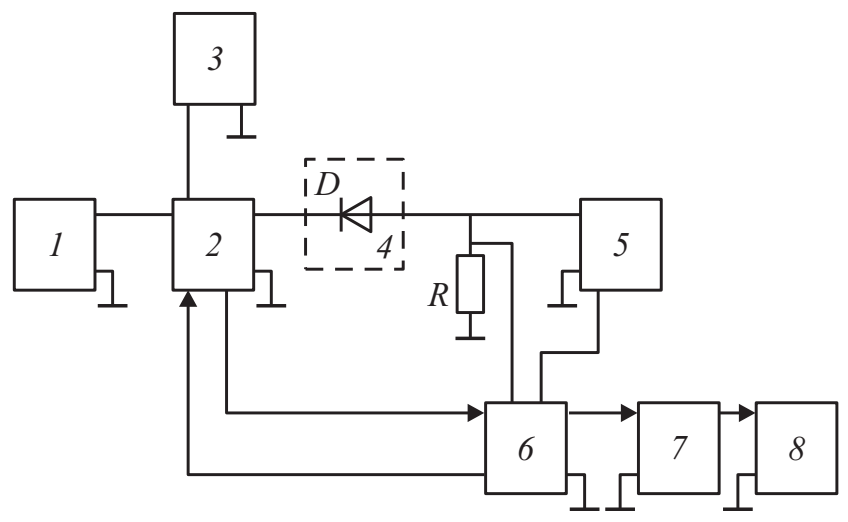

Рис. 1. Структурная схема установки для измерения задержки пробоя. 1 - регулируемый источник постоянного напряжения, 2 - генератор прямоугольных импульсов, 3 - вольтметр, 4 - криотермостат, 5 - запоминающий осциллограф, 6 - блок управления, 7 - измеритель времени, 8 - персональный компьютер, $R-$ резистор, $D-$ исследуемый диод.

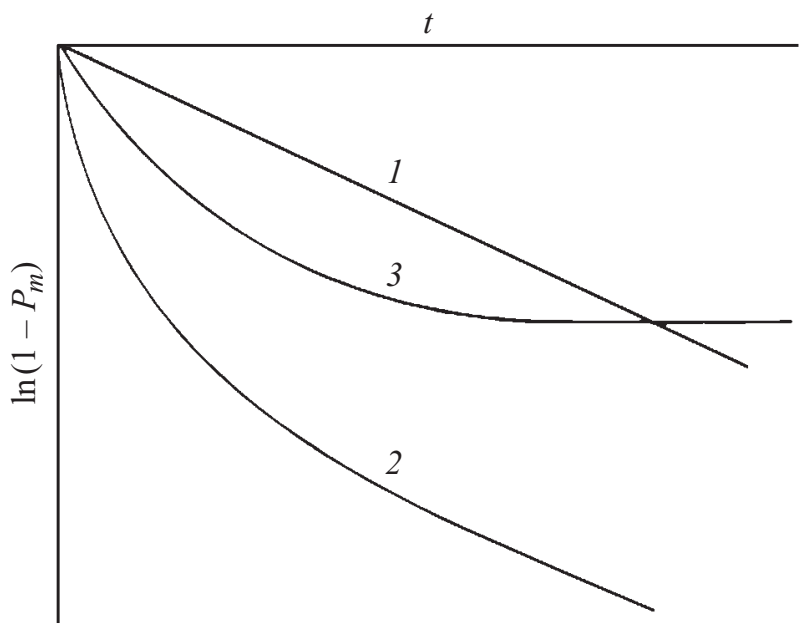

Рис. 2. Функция распределения статистической задержки пробоя по длительности. 1 - без заполнения ГЦ, $2-$ при заполнении ГЦ, 3 - разностная кривая.

ГЦ на задержку пробоя, которую можно записать в следующем виде:

$$
1-P_{M t}=\exp \left[A\left(1-\exp \left(-\frac{t}{\tau}\right)\right)\right]
$$

где

$$
A=-S_{M} N_{t} \frac{e_{n}^{2}}{\left(e_{n}+e_{p}\right)^{2}}\left[\int_{L_{m}}^{L_{n}} P_{n}(x) d x-\frac{e_{p}}{e_{n}} \int_{L_{m}}^{L_{n}} P_{p} d x\right]
$$

Если в ОПЗ $p-n$-перехода имеется несколько простых двухзарядных глубоких уровней, то процессы эмиссии носителей заряда с них будут идти независимо и функция распределения задержки пробоя в полулогарифмических координатах будет представлять собой сумму отдельных компонент. 
В реальных $p-n$-переходах наряду с эмиссией носителей заряда с ГЦ имеются и другие механизмы, поставляющие носители для запуска лавины. В этом случае правая часть выражения (2) должна быть дополнена еще одним, чаще всего линейным слагаемым. Его вклад также можно учесть при измерении функции распределения задержки пробоя без заполнения ГЦ.

\section{3. Экспериментальные результаты}

Исследование задержки лавинного пробоя проводилось на промышленных фосфидгаллиевых светодиодах АЛ341Г. Для измерений были отобраны образцы, которым были присущи все признаки „классических“ микроплазм: появление импульсов тока, характерного излома на вольт-амперной характеристике, достаточно широкий диапазон перенапряжения первой микроплазмы.

Кристалл GaP : N-светодиода представляет собой эпитаксиальную $p-n$-структуру. На монокристаллической подложке фосфида галлия $n$-типа проводимости, легированной теллуром, методом жидкофазной эпитаксии выращиваются два слоя: $n$ - и $p$-типа проводимости. Слой $n$-типа легировался азотом и остаточными донорами, $p$-слой — цинком и азотом.

Для данного $p-n$-перехода более близка модель плавного $p-n$-перехода с градиентом концентрации $a=2.1 \cdot 10^{22} \mathrm{~cm}^{-3}$. В районе металлургического перехода распределение легирующей примеси соответствует типичному диффузионному $p$-n-переходу. Стационарное напряжение пробоя первых микроплазм в различных диодах составляло 18-19 В при комнатной температуре. Напряжение включения второй микроплазмы отличалось от напряжения включения первой (при различных температурах) более чем на $1.2 \mathrm{~B}$.

Исследования проводились в диапазоне температур $300-380 \mathrm{~K}$, перенапряжение составляло $1.1 \mathrm{~B}$, а напряжение заполнения глубоких центров - 10 В.

Типичные экспериментальные функции распределения длительности статистической задержки пробоя микроплазмы представлены на рис. 3. В исследуемом диапазоне температур результирующая кривая 3 описывалась одной экспонентой или суммой экспонент вида (4). Это позволило провести определение постоянных времени установления стационарного состояния глубоких центров $\tau$. На рис. 4 приведены зависимости $\tau$ от $T$ в виде графиков Аррениуса. Найдены параметры энергетических уровней, которые приведены в таблице.

Параметры обнаруженных глубоких уровней

\begin{tabular}{c|c|c}
\hline $\begin{array}{c}\text { Обозначение } \\
\text { энергетического } \\
\text { уровня }\end{array}$ & Энергия $\Delta E_{t}$, эВ & Сечение захвата, см$^{2}$ \\
\hline$E_{1}$ & 0.52 & $1.23 \cdot 10^{-18}$ \\
$E_{2}$ & 0.41 & $9.5 \cdot 10^{-20}$ \\
$E_{3}$ & 0.60 & $1.44 \cdot 10^{-16}$ \\
$E_{4}$ & 0.68 & $2.9 \cdot 10^{-14}$
\end{tabular}

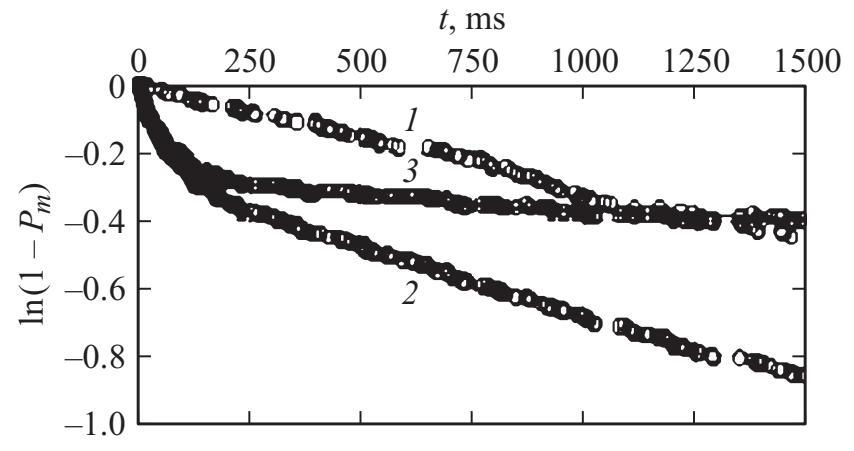

Рис. 3. Типичные кривые функции распределения статистической задержки пробоя по длительности при температуре $354.2 \mathrm{~K}: 1$ - без заполнения ГЦ, 2 - с заполнением ГЦ, 3 - разностная кривая.

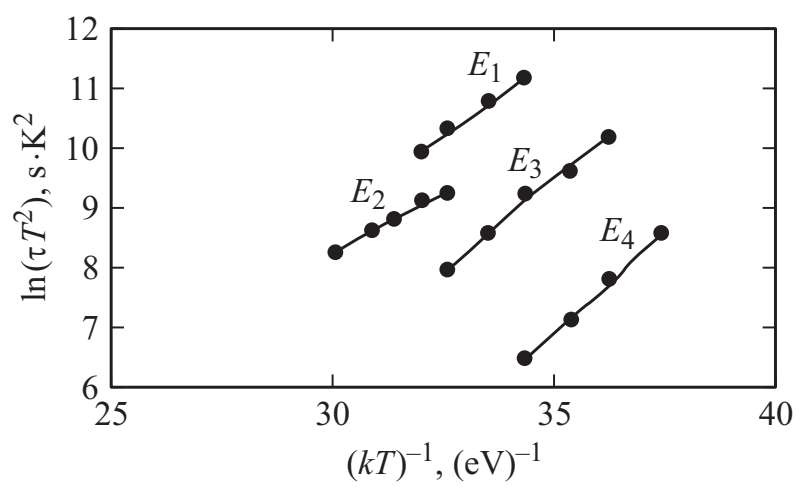

Рис. 4. Кривые Аррениуса.

При изменении зарядового состояния глубоких центров частичным снижением напряжения на $p-n$-переходе по статистической задержке микроплазменного пробоя определен энергетический спектр глубоких уровней в микроплазменных каналах $\mathrm{GaP}: \mathrm{N}$-светодиодов. В диапазоне температур 300-380 K обнаружено влияние четырех глубоких уровней. Первый с энергией 0.52 эВ и сечением захвата $1.23 \cdot 10^{-18} \mathrm{~cm}^{2}$, второй с энергией 0.41 эВ и сечением захвата $9.5 \cdot 10^{-20} \mathrm{~cm}^{2}$, третий с энергией 0.60 эВ и сечением захвата $1.44 \cdot 10^{-16} \mathrm{~cm}^{2}$, четвертый с энергией 0.68 эВ и сечением захвата основных носителей заряда $2.9 \cdot 10^{-14} \mathrm{~cm}^{2}$. Так как исследуемый $p-n$-переход имеет симметричную структуру, определить от какой зоны отсчитываются уровни не представляется возможным.

Определить концентрацию ГЦ не удалось. Для ее определения необходимо провести расчет вероятности запуска лавины носителями, стартующими из области заполнения, т.е. от границы $L_{m}$ до $L_{n}$. Вероятность запуска лавины, рассчитанная по методике [1] для линейных фосфидгаллиевых $p$-n-переходов с напряжением пробоя 18-19В, дает величину на много порядков меньшую, чем та, которая необходима для получения разумных значений концентрации глубоких центров. Таким образом, в фосфидгаллиевых светодиодах зеленого 
спектра излучения, так же как и в светодиодах красного спектра излучения [9], наблюдается аномально высокая вероятность запуска носителями лавины. Емкостными методами, в частности методом DLTS, глубокие центры в исследуемых светодиодах нами не обнаруживались. Это свидетельствует либо о низкой концентрации ГЦ, либо об их локализации в микроплазменных каналах.

Найденные значения энергии уровней $E_{2}=0.41$ эВ, $E_{3}=0.60$ эВ, $E_{4}=0.68$ эВ совпадают с уровнями, которые наблюдались в фосфиде галлия другими методами и связаны с присутствием меди $-E_{v}+0.68$ эВ, неизвестный уровень $-E_{c}-0.6$ эВ, кобальта $-E_{v}+0.41$ эВ. Уровень с энергией 0.52 эВ в фосфиде галлия не отмечается [10]. Для точной идентификации найденных уровней проведенных измерений не достаточно. Они могут принадлежать как примесным центрам, так и структурным дефектам. Не исключено, что найденные уровни проявляются лишь в микроплазменных каналах и соответственно другими методами не обнаруживаются.

\section{4. Заключение}

Изложенные выше результаты показывают, что на задержку лавинного пробоя в $\mathrm{GaP}: \mathrm{N}$-светодиодах большое влияние оказывают ГЦ. Изменение зарядового состояния глубоких центров даже при небольшом снижении обратного напряжения (примерно на 4-5 В относительно напряжения пробоя первой МП) приводит к заметному увеличению вероятности включения микроплазмы. Показано, что изменение зарядового состояния ГЦ частичным снижением напряжения на $p-n$-переходе, статистическая задержка позволяет определять параметры ГЦ, локализованные в канале микроплазмы.

Рассмотренный метод не накладывает ограничений на геометрию $p-n$-перехода и может быть использован для исследования глубоких центров в районе локализации микроплазм, когда емкостные методы становятся неприменимы.

\section{Список литературы}

[1] И.В. Грехов, Ю.Н. Сережкин. Лавинный пробой $p-n$ перехода в полупроводниках (Л., Энергия, 1980).

[2] С.В. Булярский, Ю.П. Сережкин, В.К. Ионычев. ФТП, 33 (4), 494 (1999)

[3] С.В. Булярский, Ю.П. Сережкин, В.К. Ионычев. ФТП, 33 (11), 1345 (1999).

[4] M.W. Nield, J.H. Leck. J. Appl. Phys., 18 (2), 185 (1967).

[5] K.I. Nuttall, M.W. Nield. Sol. St. Electron., 18(1), 13 (1975).

[6] G. Ferenczi. Sol. St. Electron., 17 (9), 903 (1974).

[7] В.К. Ионычев, А.Н. Ребров. ФТП, 43 (7), 980 (2009).

[8] D. Johnson, D. Pogany, J. Willemen, E. Gornik, M. Stecher. IEEE Trans. on Electron Dev. 57 (10), 2470 (2010).

[9] С.В. Булярский, Ю.П. Сережкин, В.К. Ионычев. Письма ЖТФ, 25 (5), 9 (1999).

[10] Л. Милнс. Примеси с глубокими уровнями в полупроводниках (М., Мир, 1977).

Редактор Г.А. Оганесян

\section{Investigation of deep centers in the microplasma channels fosfidgallievyh green LED radiation spectrum}

\author{
V.K. lonychev, A.A. Shesterkina \\ Mordovian State University, \\ 430000 Saransk, Russia
}

\begin{abstract}
The study of the statistical delay of microplasma breakdown fosfidgallievyh green LED emission spectrum. In the temperature range from 300 to $380 \mathrm{~K}$ revealed an unusual strong influence deep center for statistical avalanche delay when changing their charge state reduction of a reverse voltage to the $p-n$-junction. It revealed four deep level and to determine their options.
\end{abstract}

